\title{
Adaptive Gain Control for High Dynamic Range Image Display
}

\author{
Sumanta Pattanaik ${ }^{1}$ and Hector Yee $^{2}$ \\ ${ }^{1}$ Univ. of Central Florida, ${ }^{2}$ Westwood Studios
}

\begin{abstract}
Realistic display of high dynamic range images without introducing any artifact is a hard problem. In this paper we address this problem using a detail preserving local gain control approach. Unlike many other local gain control methods available in the literature, our method is simple, and does not introduce ugly "halo" artifacts around the high dynamic range edges. We demonstrate the usefulness of this method by showing several examples.
\end{abstract}

Keywords: Adaptation, local gain control, high-dynamic range image, tone mapping

\section{Introduction}

The images we perceive in our daily life normally consist of light reflected from object surfaces. This reflected light depends on the amount of light incident on the surface and on the reflectance of the surface. In general, variation in the incident illumination is slow. Hence, the change in the perceived light intensity is mostly due to the change in surface reflectance values. Though the reflectance change at the junction of dissimilar objects can lead to significant intensity change (say, 20:1 in the case of printed black letters on a white page), average changes are much less in magnitude. Such approximation regarding the visual world breaks down in situations where both lighted and shadowed areas are visible, or the light source is present in the visual field. The intensity changes across the shadow edges, or across the edge of the light source surface can be very high (> 100:1). This high intensity change coupled with variations in reflectance gives rise to high dynamic range scenes with illumination range of more than 1000:1.

In recent years, graphics community is seeing an increasing demand for capturing and usage of high dynamic range images [Debe01]. State of the art digital cameras utilizing 12 bit or wider A/D converters are able to capture this dynamic range. Software methods are also able to extract high dynamic range information from 8bit images of high dynamic range scenes captured at multiple exposures [Debe97, Naya00]. Unfortunately, dynamic range of image display devices and image display mediums are slow in catching up with the progress in digital image capture devices and methods. The dynamic range available for image display is between only one and two orders of magnitude. Simple scaling to fit the high dynamic range image data to the range of the available displays results in loss of image detail (local contrast) in bright areas and in dark areas. The detail in darker areas become indistinguishable from black and detail in brighter areas become indistinguishable from white.

In this paper, we present a very simple method for accurately displaying such images. Using a local adaptation algorithm our method compresses high intensity changes while preserving intensity changes due to the change in surface reflectance with minimal compression. We detect the presence of high contrast edges and remove the influence of intensities present across the high contrast edge from the gain control. We demonstrate the effectiveness of our algorithm by applying it to a number of high dynamic range images.

\section{Background}

Significant advances have been made in accurately displaying low dynamic range scenes under a wide range of ambient illumination conditions [Tumb93, Ward94, Ferw96, Patt00]. Most of these algorithms attempt to simulate the adaptation behavior of the human visual system to the ambient illumination of the scene. Though individual display algorithms differ from each other in detail, a typical algorithm operates by deriving a scale factor (gain) dependent on the scene ambient illumination and then by multiplying (gain control) this scale factor with each pixel to produce the display pixel value. The resulting image preserves the relative changes in intensity due to the change in reflectance.

However, such uniform scaling approach does not work for high dynamic range scenes. The main reason of this failure is: A single ambient is not representative of the whole scene. The visual system adapts locally to the prevailing illumination condition [Patt98] and this illumination condition is likely to be very different from region to region in a high dynamic range scene.

There have been a number of attempts to devise a local gain control (adaptive scaling) method for displaying high dynamic range scenes [Chiu93, Patt98, Rahm96, Tumb98, Tumb99, Ward97]. Most of these techniques provide a form of local gain control by computing the local ambient for each pixel from the mean of pixel values in its neighborhood. Some of these methods use weighted mean with weights decreasing as a

\footnotetext{
${ }^{1}$ Dept. of Computer Science, CSB-250, UCF, Orlando, FL-32816-2362, email:sumant@cs.ucf.edu

${ }^{2}$ Westwood Studios, Los Vegas, Nevada. email: hectoryee@westwood.com
} 
(a)

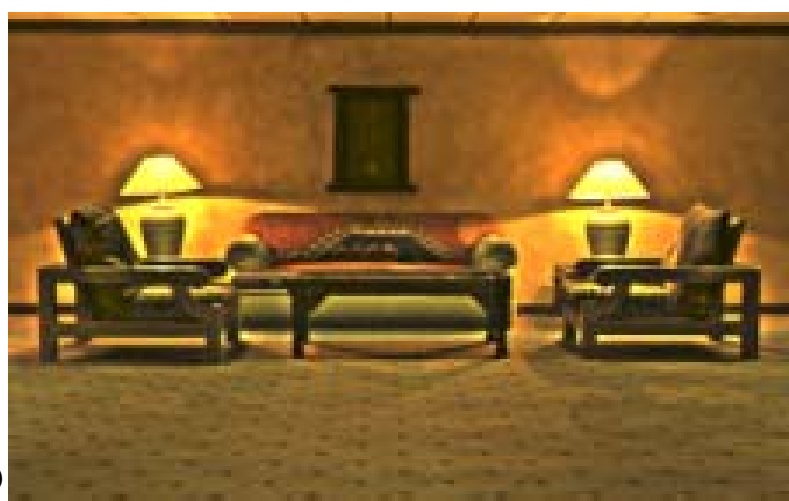

(b)

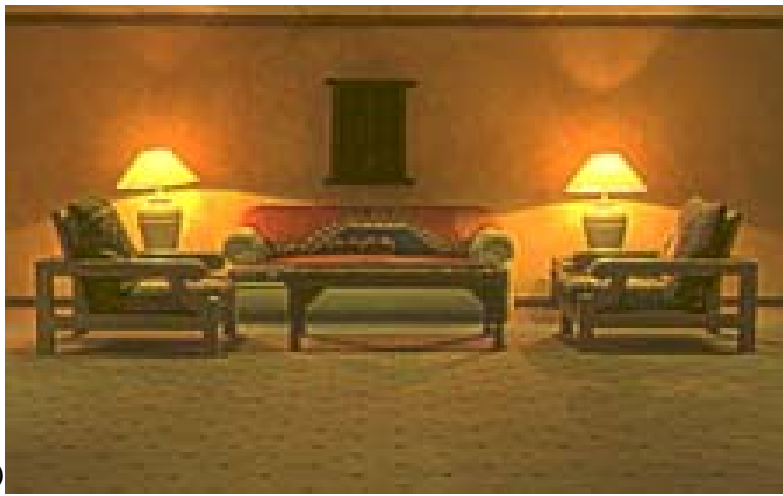

Figure 1: Local gain control using (a) local ambient computed from the local mean (b) local ambient computed bv our algorithm.

function of distance. While these methods are able to compress the dynamic range, they invariably introduce artifact (in the form of halos) around the high contrast edges (the boundaries which separate bright areas from dark areas, for example, see the region around the lamp shades). The image in Figure 2(a) highlights this problem.

The image in Figure 2(a) has been computed using the mean value of the pixels in a small neighborhood around each pixel as the local ambient value for adaptive gain control. This simple method compresses the dynamic range while preserving the details. However, the halos introduced around the high contrast edges reduce the quality of the image significantly (see the dark halo around the lamp shades). These halos arise because on the dark side of the high contrast edge the local mean becomes significantly larger due to the influence of the bright neighboring pixels. This large mean value underestimates the gain and hence the pixels after gain control look darker. Similarly, for the pixels on the brighter side of the high contrast edge, the local mean overestimates the gain, and thus makes the gain controlled pixels brighter than expected. Researchers have attempted to reduce the spread of this halo by using weighted mean values as ambient for local gain control [Rahm96, Patt98]. Single scale Gaussian function or sum of multiple scale Gaussian functions has often been used as the weight function. The drop-off in the Gaussian weight as a function of distance reduces the contribution of pixels away from the center and hence reduces the influence of farther away extreme pixels. This approach only reduces the severity of the artifact, but does not completely remove them.

The key to removing this problem is to remove the influence of extreme pixels values from the gain control process. Though the exact detail of the method developed by Tumblin et al [Tumb99] is different from the method discussed in the earlier paragraphs, to our knowledge his is the only work based on local gain control by avoiding the influence of extreme pixels. He developed a method called low-curvature image simplifier (LCIS) to reduce the scene to many smooth regions bounded by the high contrast edges. This simplified scene was used for gain control and produced display images without any artifact. Unfortunately the method is complex and computation intensive, and hence is not widely used.

In the next section we present our method for local gain control, which uses a simple and effective method for avoiding the influence of extreme pixels from the local ambient at the high intensity boundaries. Image in Figure 2(b) is computed using our gain control method. Note that the dynamic range compression is similar to the compression in Figure 2(a). However, the absence of halos significantly improves the quality of the image.

\section{The Method}

The main goal of our method is to preserve detail in both lighted and dark areas. We achieve this goal by local gain control. For every pixel, gain is derived from a local ambient. The weighted mean of pixels in a local neighborhood is used as this local ambient. Unlike many other methods already available, our weight function is independent of the position of the neighboring pixels. But, it is dependent on the magnitude of the change in intensity of the neighboring pixels from the pixel under consideration. The weight function is derived in the following paragraphs.

To avoid the influence of extreme pixels at high intensity boundaries, we wish to use only those neighboring pixels whose intensity variation from the pixel under consideration are within a factor of 5 . We place a circular mask around every pixel (intensity $I_{c}$ ) and compute the geometric mean of only those pixels (intensities $I$ ), which are within the circular mask and satisfy the following condition:

$$
\begin{array}{cl} 
& \frac{1}{5} \leq \frac{I}{I_{c}} \leq 5 \\
\text { or } & -1 \leq \log _{5}(I)-\log _{5}\left(I_{c}\right) \leq+1 \\
\text { or } & \left|\log _{5}(I)-\log _{5}\left(I_{c}\right)\right| \leq 1
\end{array}
$$

The value 5 was chosen based on the standard convention used in the field of photography, of white being 5 times as intense as mid grey and black being a little less than $1 / 5^{\text {th }}$ as intense as mid grey [Hunt95].

We use the mean intensity as the local ambient for the center pixel and compute its gain from any of the number 
of equations available in the literature [Ward94, Ferw96 or Patt00]. Choosing pixels for mean computation based on the condition defined in Equation 1 is equivalent to computing a weighted mean with the weight function defined in Equation 2.

$$
\text { weight }(I)=\left\{\begin{array}{cc}
1 & \text { if }\left|\log _{5}(I)-\log _{5}\left(I_{c}\right)\right| \leq 1 \\
0 & \text { otherwise }
\end{array}\right.
$$

Use of such weighted mean as local ambient, will suffice for images with sharp boundaries. However, photographs of natural scenes tend to have boundaries with a slightly smoother profile. In order to accommodate for slightly smooth high intensity boundaries we choose a smoother form of Equation 2. The smooth function is given in Equation 3.

$$
\text { weight }(I)=e^{-\left|\log _{5}(I)-\log _{5}\left(I_{c}\right)\right|^{25}}
$$

Figure 2 plots of this function. Like Equation 2, this function evaluates very close to 1 almost everywhere except at the boundaries of the cut-off range, where the function smoothly falls to zero.

Smith et al [Smit97] have developed an efficient edge detection algorithm using a similar equation. The value of 25 as the exponent in Equation 3 allows for a smooth but almost sharp drop off in the function from one to zero. Any value close to 25 may be equally acceptable.

The MATLAB implementation of this algorithm is given in the appendix. We directly use the function defined in Equation 3 to compute the weighted geometric mean of the neighboring pixels.

The computational complexity of this algorithm is not very different from the complexity of the algorithms using a gaussian filter. The only additional overhead is the computation of weight of the filter at every pixel.

Though we have not tried to do so, the algorithm may be optimized by:

- using look tables for the computation of weight, and

- avoiding the weighted mean computation in areas where neighborhood pixels are well within the required range.

The use of geometric mean instead of arithmetic mean as the local ambient has been suggested earlier by Tumblin et al [Tumb99]. We also believe that geometric mean helps in better preserving the relative ratios (local contrast) in the scene.

\section{Results}

We have successfully applied this algorithm to a number of high dynamic range images.

In Figure 1(b) we show the result obtained by applying our algorithm to a high dynamic range image (in 16 bit format) available from Columbia University web site ${ }^{3}$. The image shows that our algorithm is able to

${ }^{3}$ (http://www.cs.columbia.edu/CAVE/tomoo/RRHomePa ge/rrgallery.html)

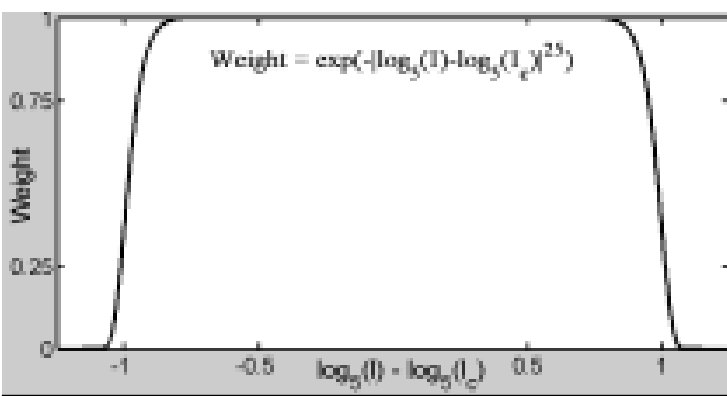

Figure 2: Weighting function used for the local ambient computation.

compress the high dynamic range without introducing any ugly artifact.

In Figure 3 we show the images obtained by applying our algorithm to high dynamic range scenes used by previous researchers in the field. Figure 3(a) shows the "parking lot" image from the scene used in [Patt98], Figure 3(b) shows the "memorial church" image from the scene used in [Ward97], and Figure 3(c) shows the "room with lamp" image from the scene used in [Chiu93].

The images in Figure 4 are processed from high dynamic range images captured using Panoscan digital camera ( $3 \times 16$ bits per color channel, from Spheron VR). These images show more detail in the brighter areas than the ones obtained by uniform scaling (Figure 5).

For all the images shown in this paper, we have used a circular window 7 pixels wide and the gain control algorithm described in [Patt00]. This algorithm requires ambient illumination specified in actual luminance values. The magnitudes of RGB values in all of the images used do not provide exact luminance values. So we scaled each image in such a way that the maximum luminance will approximately match the maximum luminance measured by the authors for a similar scene (using a spectrophotometer). The MATLAB implementation of the gain control algorithm has been made available by the authors of [Patt00] in the SIGGRAPH 2000 proceedings CD. The original code uses a single (global) ambient luminance value for the whole image. We modified the code to accept local ambient luminance for every pixel.

\section{Conclusion}

We have described a simple method for realistic rendition of digitally captured high dynamic range images. Unlike other local gain control methods, our method uses intensity dependent weight function for local ambient computation. Any high dynamic range image acquisition system can make use of this method. A few example applications are: digital photography, medical imaging, and aerial imaging. High dynamic range raw image data from most of the currently available consumer grade digital cameras can be offline processed and displayed using our algorithm. The 
algorithm is based on a simple convolution operation, and hence can be built into the camera hardware to directly produce 8-bit images without any loss of detail for high dynamic range scenes. Medical imaging and aerial imaging techniques often produce high dynamic range gray scale images. The visualization tools used for displaying such images will benefit from the described method by compressing dynamic range without sacrificing the visual details.

\section{Acknowledgements}

We are thankful to Peter Shirley for making the Room with Lamp image available for use, to Greg Ward for making the Memorial Church image available through his web page and to Panoscan for providing us with the sample high dynamic range panorama images used in Figure 4.

\section{References}

[Chiu93] Chiu, K., Herf, M., Shirley, P. Swamy, S., Wang, C., \& Zimmerman, K. "Spatially non-uniform scaling functions for high contrast images, Proceeding of Graphics Interface, 245-254. 1993.

[Debe97] Debevec, P., Malik, J. "Recovering High Dynamic Range Radiance Maps from Photographs", Proceedings of Siggraph 1997, Los Angeles, 369378, August 3-8 1997.

[Debe01] Debevec, P. "Image Based Lighting", Siggraph 2001 Course notes, Los Angeles, 12-17 August 2001. (http://www.debevec.org/IBL2001/)

[Ferw96] Ferwerda, J.A., Pattanaik, S., Shirley, P., and Greenberg, D.P. "A Model of Visual Adaptation for Realistic Image Synthesis", Proceedings of SIGGRAPH'96, pp 249-258, New Orleans, 4-9 August 1996.

[Hunt95] Hunt, R. W. G., The Reproduction of Color, Fountain Press, England, 1995.

[Naya00] Nayar, S. K. and Mitsunaga, T. "High Dynamic Range Imaging: Spatially Varying Pixel Exposures" Proceedings of IEEE Conference on Computer Vision and Pattern Recognition, South Carolina, June 2000.

[Patt98] Pattanaik, S. N., Ferwerda, J.A., Fairchild, M. D. and Greenberg, D. P. "A Multiscale Model of Adaptation and Spatial Vision for Realistic Image Display", Proceedings of SIGGRAPH'98, pp 287298, Orlando, July 1998.

[Patt00] Pattanaik, S. N., Tumblin, J. E., Yee, H., Greenberg, D. P. "Time-Dependent Visual Adaptation for Realistic Real-Time Image Display", Proceedings of SIGGRAPH 2000, pp. 47-54, New Orleans, 23-28 July, 2000.

[Rahm96] Rahman, Z., Jobson D., Woodell, G. "Method of Improving A Digital Image as a Function of Its Dynamic Range" US Patent \#5991456.

[Smit97] Smith, S. M. and Brady, J. M. "SUSAN - a new approach to low level image processing." International Journal of Computer Vision, 23(1):45-
78, May 1997.

(http://www.fmrib.ox.ac.uk/ steve/susan/)

[Tumb93] Tumblin, J. and Rushmeier, H. "Tone reproduction for realistic images". IEEE Computer Graphics \& Applications, 13(6), pp. 42-48, 1993.

[Tumb98] Tumblin, J., Hodgins, J. K. and Guenter, B. K. "Two method of Display of High Contrast Images", ACM-TOG.

[Tumb99] Tumblin, J. and Turk, G. "LCIS: a boundary hierarchy for detail-preserving contrast reduction" Proceeding SIGGRAPH '99, 83-90, 1999.

[Ward94] Ward, G.. "A contrast-based scale factor for luminance display". Graphics Gems IV, Academic Press Professional, pp 415-421, 1994.

[Ward97] Ward-Larson, G., Rushmeier, H., and Piatko, C. "A Visibility Matching Tone Reproduction Operator for High Dynamic Range Scenes" IEEE Transactions on Visualization and Computer Graphics, 3(4):291-306, October 1997.

\section{Appendix}

The following MATLAB program has been used to generate the images shown in this paper.

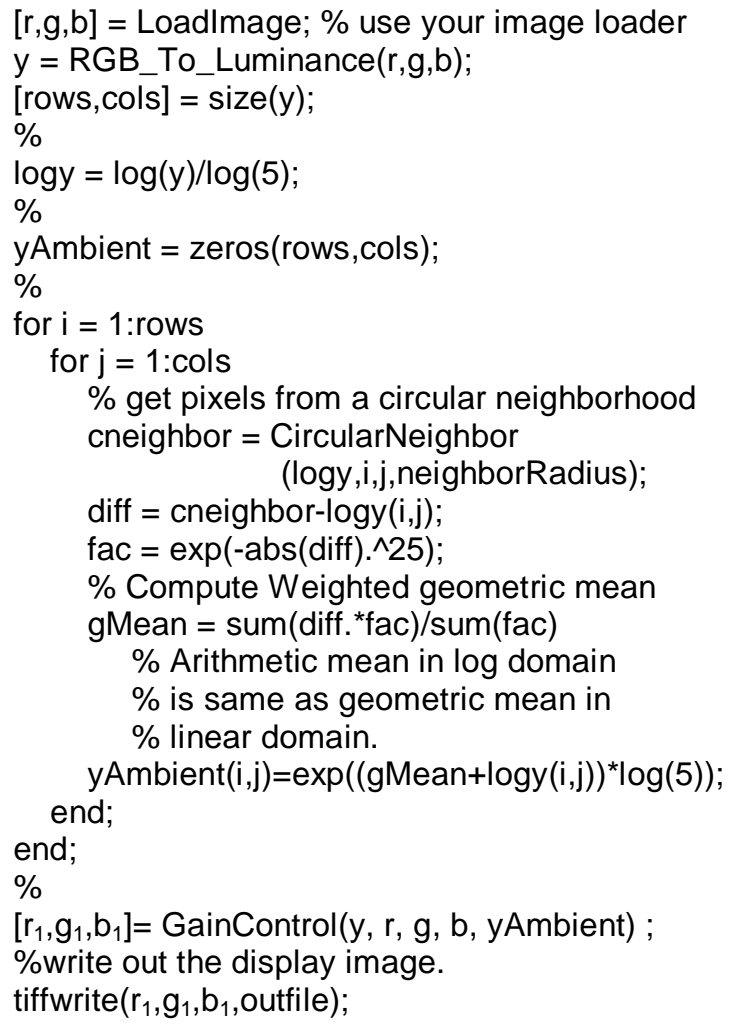




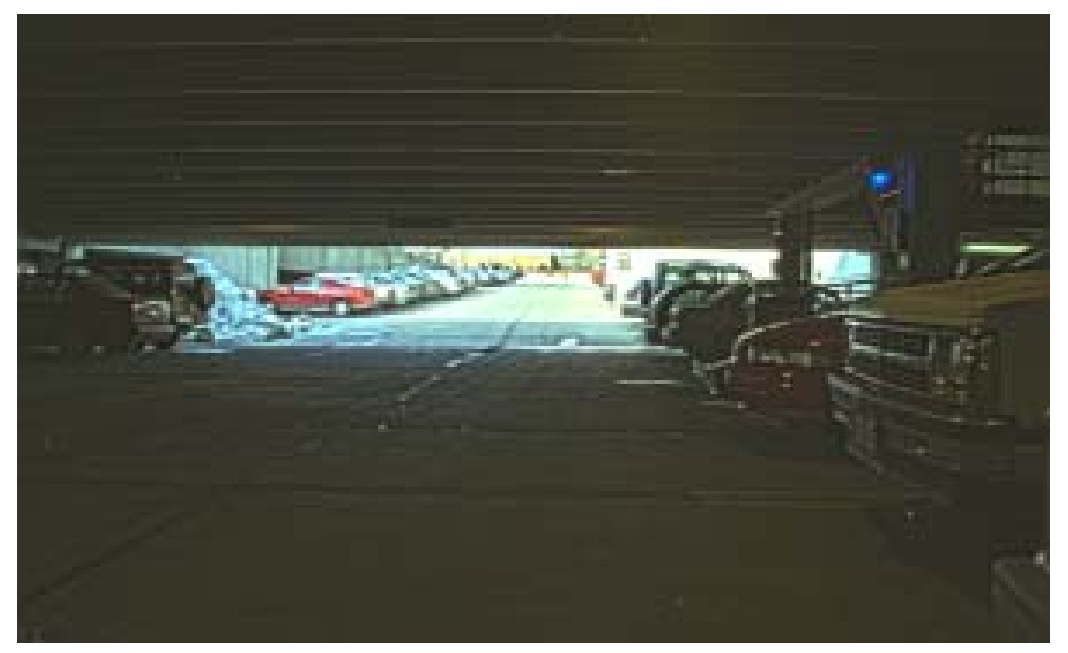

Figure 3(a)

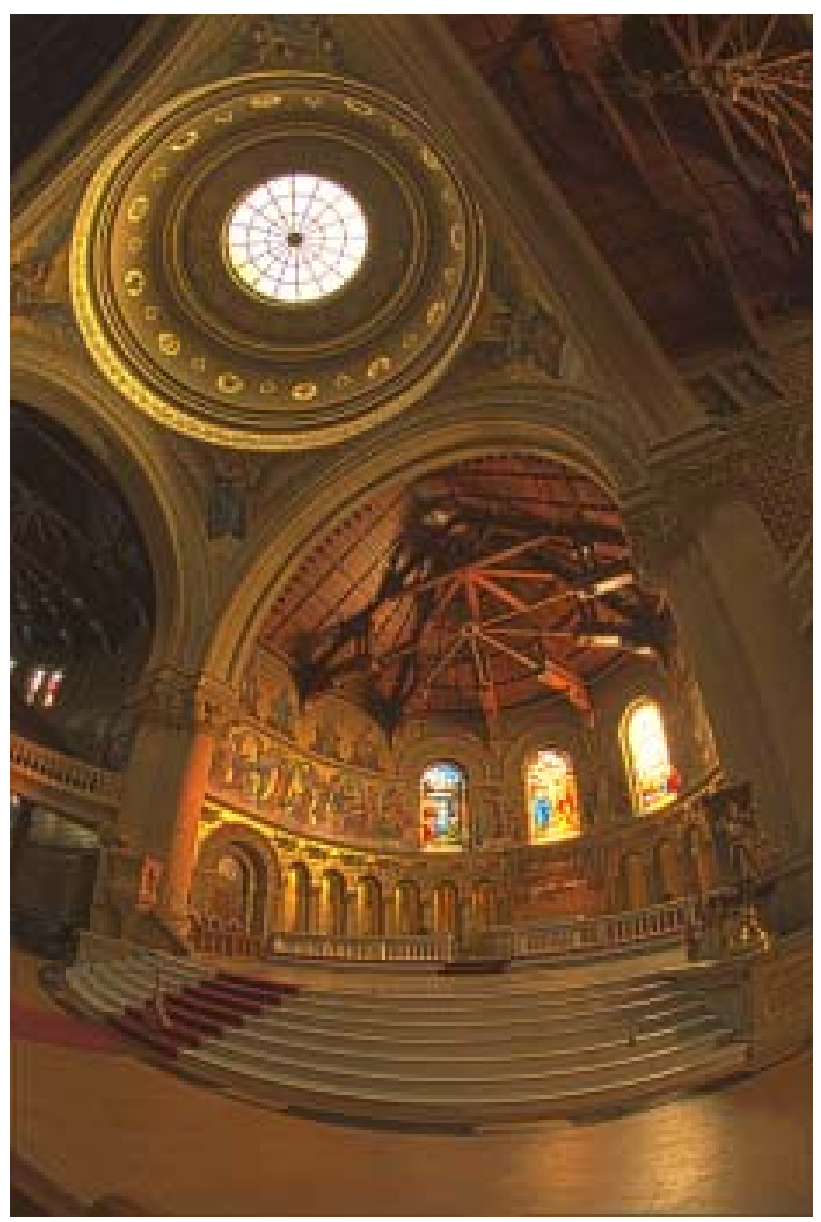

Figure 3(b)

Figure 3(c)

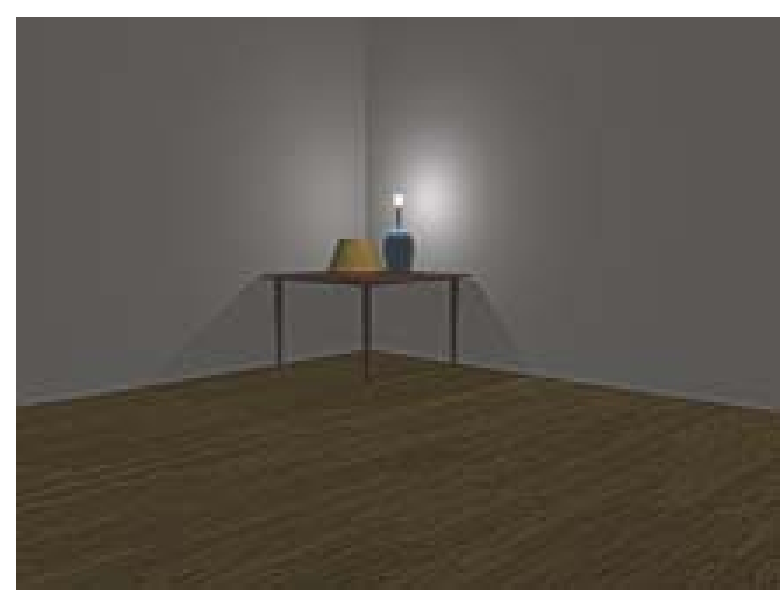

Figure 3: Images computed using our algorithm. (a) "Parking lot" image (compare with results from [Patt98]) (b) "Memorial church" image (compare with results from [Ward97]) (c) "Room with lamp" image (compare with the results from [Chiu93]). 
Figure 4(a)

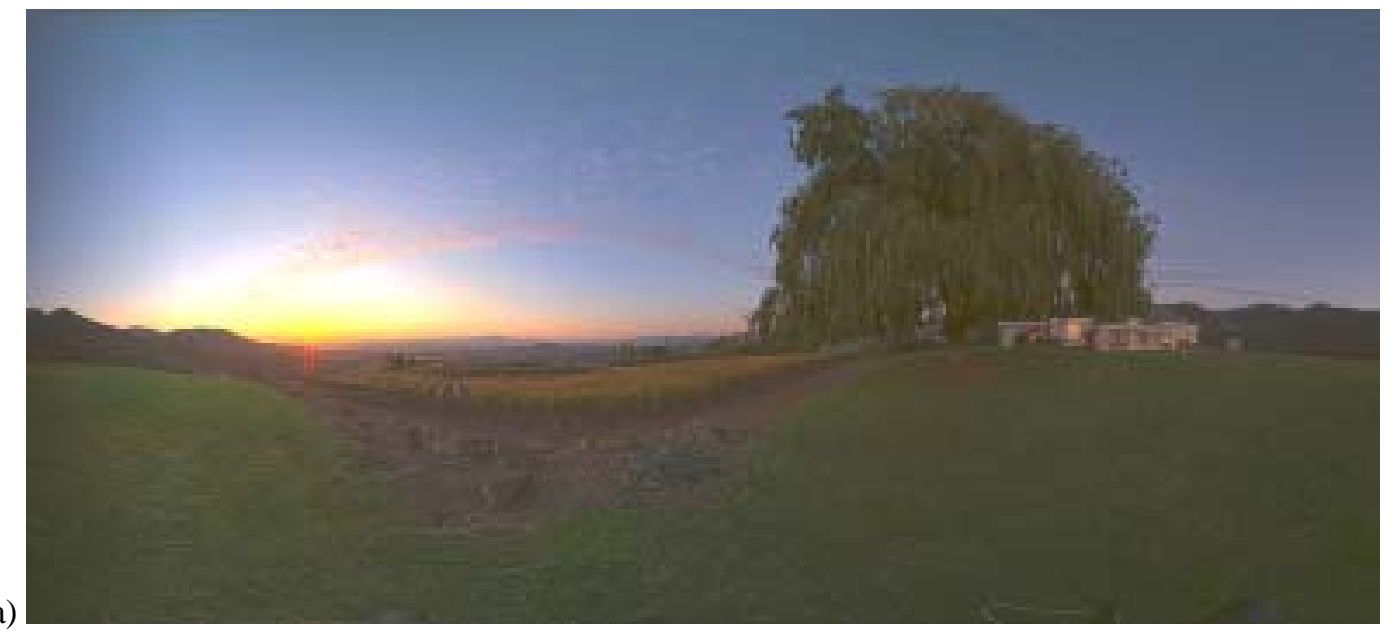

Figure 4(b)
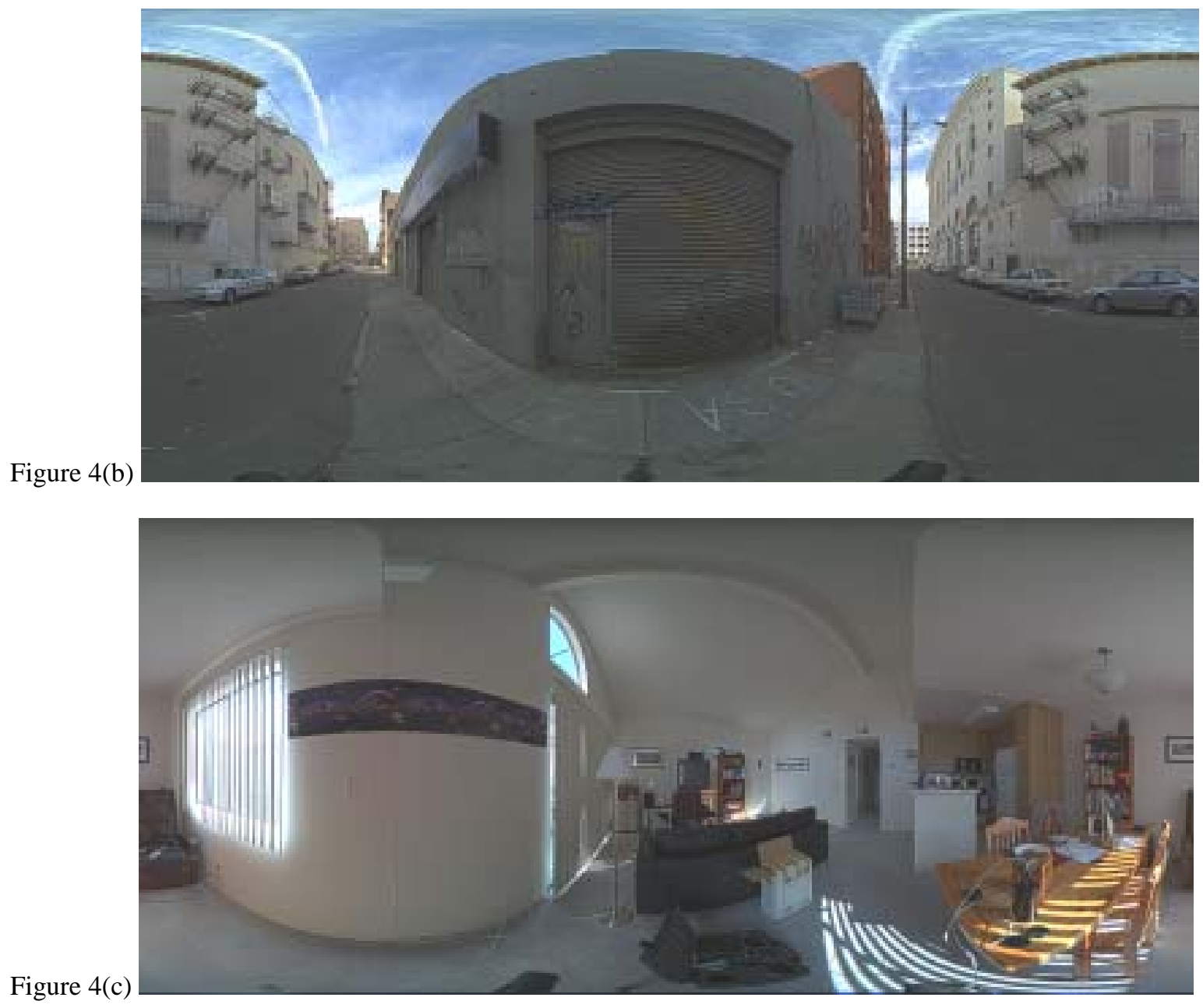

Figure 4: Panoroma images of (a) Sunset, (b) Alley, and (c) Interior of a room with sunlight. Images were captured at 16 bits per color channel using Panoscan High Dynamic Range Digital Camera. Original images were in RGBE format and were taken from a promotional CD available from www.spheron.com. Compare with the uniformly scaled versions of these images shown Figure 5. 


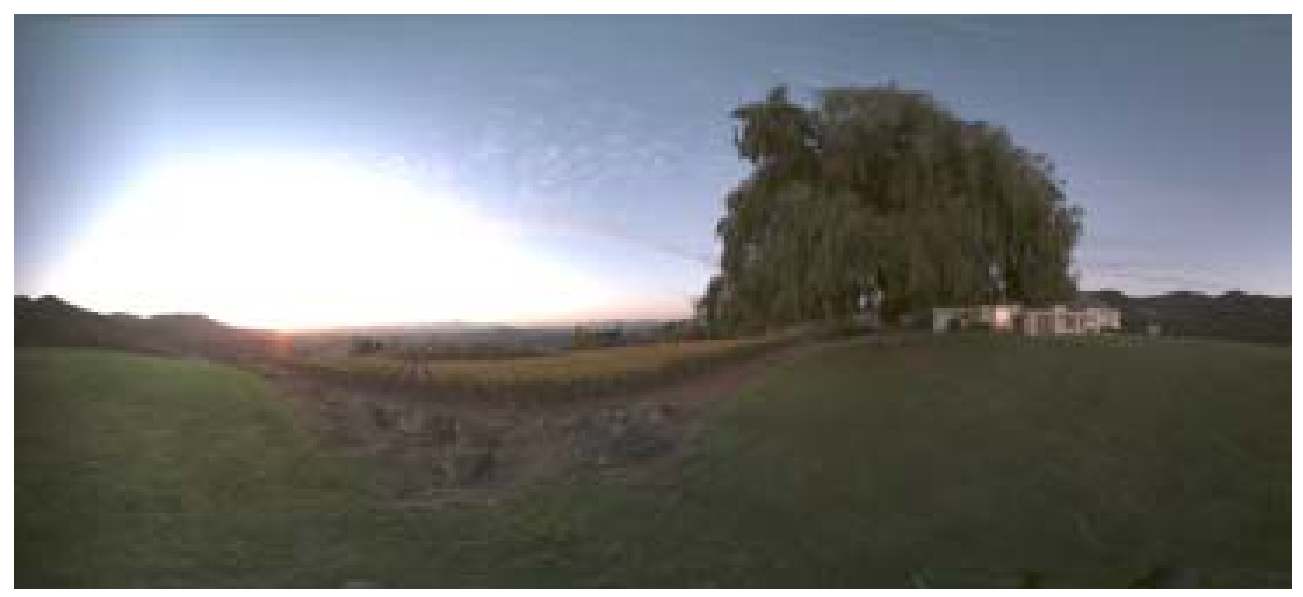

Figure 5(a)

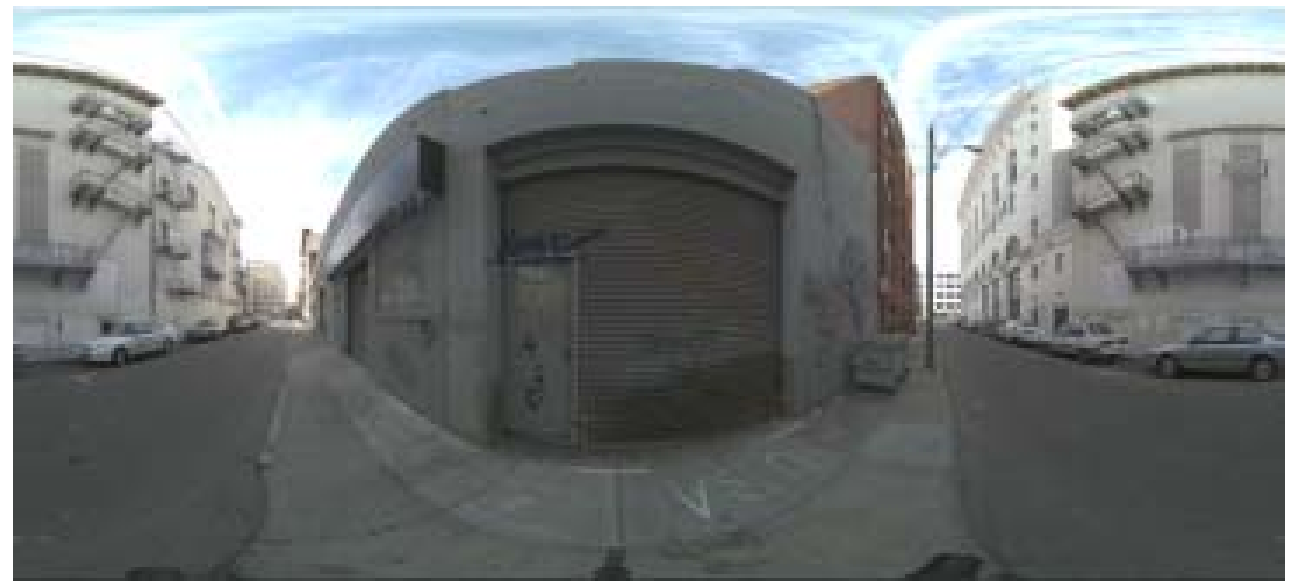

Figure 5(b)

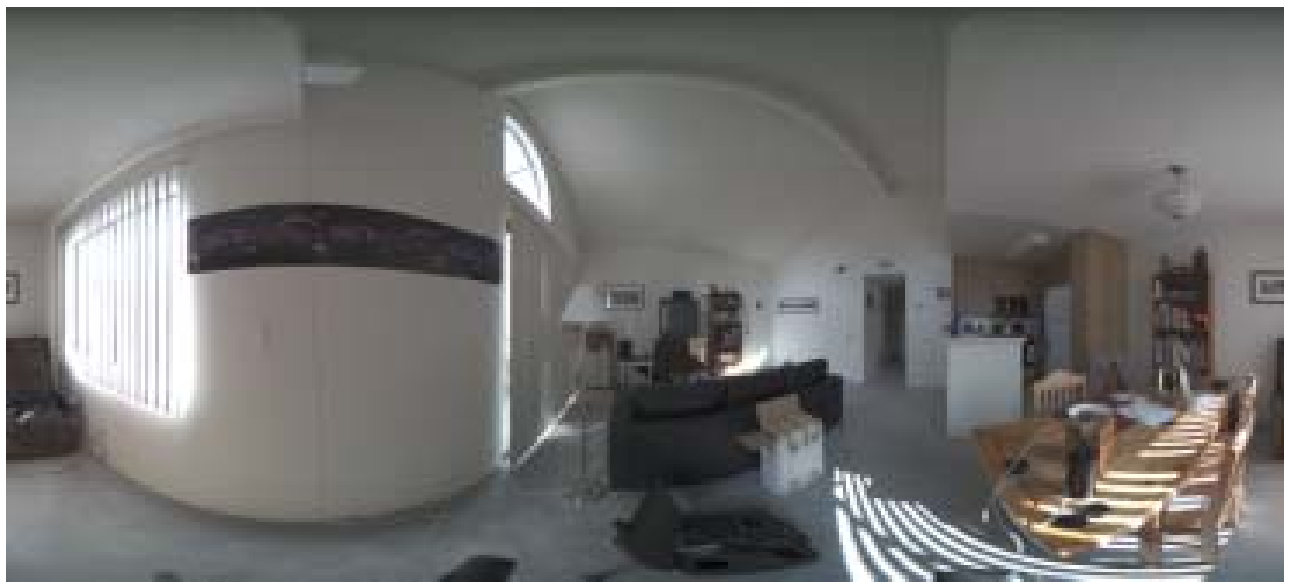

Figure 5(c)

Figure5: Uniformly scaled versions of the scenes shown in Figure 4. 